\title{
Evaluation of Dynamic / Thiol Disulfide Balance and İschemia Modified Albumin in Children with Trauma
}

\author{
CAN OZTORUN ${ }^{1}$, HAYAL DORUK ${ }^{2}$, DOĞUŞ GÜNEY ${ }^{1}$, ÜLFET NIHHAL İRDEM \\ KÖSE ${ }^{2}$, TUĞBA ÖRNEK DEMIR ${ }^{2}$, VILDAN SELIN ÇAYHAN², SABRI DEMIR ${ }^{2}$, \\ Ahmet ERTÜRK ${ }^{2}$, ALI GÜNGÖR ${ }^{2}$, AYSUN KARA UZUN², CEMIL NURAL ${ }^{1}$, Salim \\ Neselioglu $^{3}$, Mujdem Nur Azili ${ }^{1}$, and EMRAH SENEL ${ }^{1}$ \\ ${ }^{1}$ Ankara Yildirim Beyazit University \\ ${ }^{2}$ Ankara City Hospital \\ ${ }^{3}$ Yildirim Beyazit University Faculty of Medicine
}

February 5, 2021

\begin{abstract}
Introduction: Trauma is the most common cause of death in childhood. Tissue damage, ischemia-reperfusion injury and inflammatory response are mainly responsible for increasing serbest oxiygen radicals. In this study, we aimed to investigate the use of thiol-disulfide and ischemia-modified albumin levels as a diagnostic laboratory parameter in trauma children. Method: Of 202 children, 101 of them were hospitalized in pediatric surgical intensive care unit with trauma and 101 of others were healthy children. Levels of native thiol (-SH), total thiol (SH + SS), dynamic disulfide (SS), dynamic disulfide (SS) / total thiol $(\mathrm{SH}+\mathrm{SS})$, albumin and ischemic modified albumin (IMA) were measured from the sera of patients and healthy volunteers. For statistical analyses, SPSS 17.0 was used. Mann-Whitney U and paired correlation test were used where appropriate. p $<0.05$ was considered significant. Results: The mean age of the patients in the trauma group (Boys: 61 Girls: 40) was 7,88 years and the control group was 8,00 years. In trauma group, 86 of children were exposed to blunt trauma, 15 of children had penetrating trauma and 54 patients had multiple trauma. Surgical procedures were performed in 17 patients. In the trauma group, native thiol, total thiol, dynamic disulfide / total thiol, albumin and IMA levels were significantly lower than that of control $(\mathrm{p}<0.001)$ and their dynamic disulfide $(\mathrm{p}=0.001)$ was higher compared to the control. There was no difference thioldisulfide parametres in trauma groups sub-division as $\operatorname{surgery}(\mathrm{n}=17)$ vs. follow-up $(\mathrm{n}=84)$ groups or multiple trauma(n=54) vs. isolated organ trauma $(n=47)$ groups, or penetrating $(n=15)$ or blunt trauma $(n=86)$ groups. Conclusion: Thiol-disulfide balance and IMA levels show changes in favor of oxidative stress in children with trauma, however, it cannot be used as a laboratory marker that helps to show the system and organ affected by the trauma and to decide the surgical intervention.
\end{abstract}

\section{INTRODUCTION}

It is known that oxidative stress increases as a result of the activation of neutrophils and macrophages and overproduction of reactive oxygen species (ROS) in inflammatory events [1-3]. Damage due to ROS is prevented by enzymatic or nonenzymatic antioxidant mechanisms including super-oxide dismutase, catalase and glutathione S-trans enzyme systems, and important biological thiols such as glutathione, cysteine, homocysteine, N-acetyl-cysteine and gamma glutamine.

Thiol is an organic compound and contains a -SH group that plays a critical role in the prevention of oxidative stress in cells. SH-containing amino acid groups in proteins are primary targets of ROS. In the same medium with ROS, the -SH groups are oxidized and form reversible disulfide bonds. The loss of thiol groups is the main molecular mechanism that results in structural and functional changes in proteins. Antioxidants and in particular thiol groups that try to prevent the destructive effects of free radicals cannot maintain plasma 
and tissue levels during these interactions [4]. However, the resulting disulfide bonds can be reduced to the thiol groups by the cellular reduction effects of some antioxidants and thus the thiol/disulfide homeostasis is preserved [5]. The state of the dynamic thiol-disulfide balance plays a critical role in antioxidant protection, detoxification, signal transduction, apoptosis, the regulation of enzymatic activity, transcription factors and cellular signaling mechanisms [6]. In addition, distortions in the dynamic thiol-disulfide balance has been shown to play a role in the pathogenesis of many diseases such as diabetes, cardiovascular diseases, cancer, rheumatoid arthritis, chronic kidney disease, AIDS, Parkinson's disease, Alzheimer's disease, Friedrich's ataxia, multiple sclerosis, amyotropic lateral sclerosis and liver diseases [7]. Therefore, determination of the dynamic thiol/disulfide balance may provide valuable information regarding various normal or abnormal biochemical processes [7]. A number of studies have shown the predictive value of thiol/disulfide hemostasis in organ ischemia. However, measurement of plasma/serum thiol-disulfide balance by colorimetric method has been made possible by the method developed by a Turkish scientist for the first time in the world [7].

Ischemia-modified albumin (IMA) is a FDA-approved test among the newly investigated cardiac markers [8]. The principle of the test is based on measuring the cobalt binding capacity of albumin, leading to chemical changes in the albumin during oxidation. This new albumin molecule is also called ischemiamodified albumin. The formation of this new albumin molecule, which has lost its ability to cobalt, is one of the earliest markers of ischemia [9]. However, recent studies suggest that IMA, which stands out as a marker of cardiac ischemia, may increase in different pathologies [10-12].

Trauma is one of the causes of admission to the emergency department and is the most common cause of death in childhood [13]. Both our country and the United States, trauma locates in the second place after the infectious diseases in the applications to the emergency services [14]. Trauma management should be fast and dynamic because it has a high mortality and morbidity. In all traumas, $\mathrm{ABC}$ algorithm should be started from the first encounter. The most common cause of trauma is traffic accidents. This is followed by fall from height. In children, blunt abdominal trauma is the most common trauma of the spleen and liver. Penetrating traumas in children constitute $10 \%$ of all traumas. However, mortality is higher in these patients. Gunshot wounds and sharp stab wounds are the most common causes. In the penetrating injuries in children, the most damaged abdominal organs are liver $(28.8 \%)$, bowel $(26 \%)$, abdominal large vessels (24.9\%), diaphragm (22\%), kidneys (11.3\%), spleen (9.6\%) and esophagus (9.6\%), respectively [15].

\subsection{Trauma and Oxidative stress}

Trauma leads to oxidative stress by increasing the production of SORs and SARs due to the activation of polymorphonuclear cells and neutrophils stemming mainly from tissue damage, ischemia-reperfusion injury and consequently inflammatory response. It is known that oxidative stress is increased as a result of the activation of neutrophils and macrophages and over-production of SOR in inflammatory events $[16,17,18]$. In inflammation, SORs produced by polymorphonuclear leukocytes lead to lipid peroxidation in cell membranes. These changes in the cell membrane cause an increase in microvascular permeability, intestinal edema, infiltration of inflammatory cells, activation of neutrophils and cell death [17]. In literature, it has been shown in studies conducted by adult patients and animals that the head injury [19-22], burn [23], bone fractures [24], liver damage [25], kidney damage [26], lung injury [27] and similar traumas lead to distortion in oxidative balance resulting in oxidative stress. However, there are very few studies in children [28]. In traumatized children, the development of a test that could show the affected system and organ from trauma more quickly and easily and assist in deciding on surgical intervention would help both early diagnosis and early surgical intervention. In this study, we aimed to investigate the use of thiol-disulfide and ischemiamodified albumin levels as a diagnostic laboratory parameter in trauma children.

\section{MATERIAL and METHODS}

Of 101 children who were hospitalized between $1^{\text {th }}$ April 2017 and $1^{\text {th }}$ June 2018 in pediatric surgical intensive care unit due to trauma and 101 other children admitted by circumcision or incarcerated inguinal hernia were included to this study. The study was conducted prospectively in our hospital. The study permission was obtained from the ethics committee of the Ankara Child Health and Diseases Hematology Oncology Training 
and Research Hospital (decision no. 2017/05 dated 27/03/2017). Informed consent form was obtained from the parents of both groups' children included to the study. The cases with trauma were compared with the control group in terms of thiol-disulfide levels and IMA. On the 2nd and 5th days of hospitalization, $2 \mathrm{~mL}$ of venous blood was taken to the yellow blood tube (BD Vacutainer plastic SST II tube $($ ) ) from children with trauma. Blood samples collected were centrifugated at $3600 \mathrm{rpm}$ for 10 minutes in the biochemistry laboratory and $2 \mathrm{cc}$ serum was obtained and stored at $-80^{\circ} \mathrm{C}$. After all the samples were collected, all of them were thawed at the same time and the blood thiol-disulfide parameters were determined by Erel \& Neselioglu with the newly developed automatic measurement method [7] and the IMA levels were determined by Bar-Or et al. [29] method via the Roche Hitachi Cobas c501 automatic analyzer. The levels of native thiol (-SH), total thiol $(\mathrm{SH}+\mathrm{SS})$, dynamic disulfide (SS), dynamic disulfide (SS) / total thiol $(\mathrm{SH}+\mathrm{SS})$, albumin and ischemic modified albumin (IMA) were measured from the sera of patients and control group.

2.1.Statistical analysis: Statistical analysis was performed using SPSS 17.0 version (Chicago Inc., 2008). Shapiro-Wilk test was used to determine normality of the continuous variables. Numerical variables were controlled by analytical methods. Total thiol, native thiol (SH), dynamic disulphid (SS) and dynamic disulphid (SS) / total thiol (SS + SH), albumin and IMA values did not fit the normal distribution. For comparison, variables were analyzed by Mann-Whitney $\mathrm{U}$ and paired correlation test. $\mathrm{p}<0.05$ was considered significant.

\section{RESULTS}

In this study, 101 children with trauma were evaluated and their variables were compared with 101 healthy children.

\subsection{Demographics of the groups}

The mean age of the patients in the trauma group (boys: 61 girls: 40) was 7.8 years (standard deviation $=$ 4.77 , range $=0.8-17$ years), and the control group 8 years (standard deviation $=3.9$, range $=1-17$ years). 86 of the cases in the trauma group were exposed to the blunt force, 15 to penetrating trauma, and 54 patients had multiple trauma. Surgical procedures were performed in 17 patients. The organs and systems of the cases affected by trauma were shown in Table 1 . The most commonly affected organ was the spleen $(\mathrm{n}=$ $37)$.

\subsection{Laboratory findings}

The trauma and control groups' blood parametres of native thiol $(\mathrm{SH})$, total thiol $(\mathrm{SH}+\mathrm{SS})$, dynamic disulfide (SS), dynamic disulphid (SS) / total thiol (SS $+\mathrm{SH}) \%$, ischemic modified albumin (IMA) and albumin levels were shown in Table 2 .

In the trauma group, native thiol $(\mathrm{p}<0.001)$, total thiol $(\mathrm{p}<0.001), \%$ dynamic disulfide/total thiol $(\mathrm{p}$ $<0.001)$, albumin $(\mathrm{p}<0.001)$ and IMA $(\mathrm{p}<0.001)$ The decrease in the levels of 0.01$)$ compared to the control group and the increase in dynamic disulfide $(\mathrm{p}=0.001)$ compared to the control group were statistically significant.

The cases in the trauma group were divided into two subgroups as surgery $(\mathrm{n}=17)$ and follow-up $(\mathrm{n}=84)$. This two groups' native thiol, total thiol, dynamic disulfide, disulfide / total thiol ratio, albumin and IMA levels were compared (see Table 3). No statistically significant difference was found between two subgroups in terms of their thiol-disulfide parametres.

The cases in the trauma group were also divided into two other sub-groups as multiple trauma $(\mathrm{n}=54)$ and isolated organ trauma $(\mathrm{n}=47)$. Two sub-groups were also similar in terms of their native thiol $(\mathrm{p}=0.575)$, total thiol $(\mathrm{p}=0.794)$, dynamic disulphide $(\mathrm{p}=0.584)$, disulfide / total thiol ratio $(\mathrm{p}=0.414)$, albümin $(\mathrm{p}=0.792)$ and IMA ( $\mathrm{p}=0.913)$ levels (there was not table for this findings)

Lastly, the cases in the trauma group were divided into two sub-groups as penetrating $(\mathrm{n}=15)$ and those who were exposed to blunt trauma $(\mathrm{n}=86)$. Among two groups were not statistically different for native thiol $(\mathrm{p}=0.65)$, total thiol $(\mathrm{p}=0.78)$, dynamic disulfide $(\mathrm{p}=0.41)$, disulfide / total thiol ratio $(\mathrm{p}=0.70)$, albumin $(\mathrm{p}=0.14)$ and IMA $(\mathrm{p}=0.98)$ levels (see Table 4$)$. 
There was no difference between native thiol, total thiol, dynamic disulfide, disulfide / total thiol ratio, IMA, albumin levels and systems affected or organs (for all variables, $\mathrm{p}>0.05$ ). There was also no difference between 2nd day of hospitalization and 5th day of hospitalization blood thiol-disulfide parametres tested with paired correlation test (for all, $\mathrm{p}>0.05$ ).

\section{DISCUSSION}

Free radicals stemming mainly from post-traumatic processes leads to damage in lipids, proteins in the cell membrane and DNA resulting in infections, multiple organ failure, acute failure of respiratory, renal and cerebral systems [30,31]. Therefore, it is extremely important to know the levels of thiol to reduce free oxygen radicals, prevent harmful effects, and perform appropriate interventions to the patient after trauma. Oxidative stress is an indication of tissue damage from increased reactive oxygen species. Thiol inhibits cellular and tissue damage by inhibiting the effects of reactive oxygen species by lipid metabolism of the cell membrane.

It is known that oxidative stress increases after physical injuries in trauma patients. Although many studies have shown that reactive oxygen species in trauma increase and antioxidant levels to be decreased [30,32, 33], there are two studies in the literature showing the relationship between trauma and dynamic thiol-disulfide balance $[34,35]$. With this study, the relationship between trauma and thiol-disulfide and IMA levels in children was also shown.

In our study, total thiol, native thiol, disulfide/total thiol ratio were found to be significantly decreased significantly, as consistent with the literature and dynamic disulfide as increased in the trauma group compared to the control. IMA and albumin levels were also significantly decreased. In trauma group, however, there was not significant difference between surgery and follow-up sub-groups, or multiple and isolated trauma sub-groups, or type of trauma and systems affected or organs. These laboratory findings indicate that trauma results in a shift towards the oxidant side in favor of oxidative stress in children. However, there was no difference in trauma type, or whether there were surgery or not, multiple or isolated, systems or organs effected, and thiol/disulfide balance and IMA levels.

These results point out that thiol/disulfide balance and IMA levels cannot be used as an adjunctive marker in imaging and determining the affected system and organ from trauma during the follow-up period and deciding the surgical intervention. There was also not found statistical difference in total thiol, native thiol, dynamic disulfide, dynamic disulfide / total thiol rate, albümin or IMA levels between 2nd and 5th days of hospitalization of children with trauma. The development of a test that can help to determine the system and organ affected by the trauma more quickly and easily and can help in the decision of surgical intervention will help both early diagnosis and early surgical intervention.

\section{CONCLUSION}

In conclusion, thiol-disulfide balance and IMA levels, in accordance with the literature, show changes in favor of oxidative stress in children with trauma, nonetheless, it is seen that it cannot be used as a laboratory marker that helps to show the system and organ affected by the trauma and to decide the surgical intervention.

Conflicts of interest: none.

Ethical approval: All procedures performed in studies involving human participants were in accordance with the ethical standards of the institutional and/or national research committee and with the 1964 Helsinki declaration and its later amendments or comparable ethical standards.

Informed consent: Informed consent was obtained from all individual participants included in the study REFERENCES

1. Bolukbas C, Bolukbas FF, Horoz M et al. Increased oxidative stress associated with the severity of the liverdisease in various forms of hepatitis B virus infection. BMC Infect Dis (2005) 31: 5-95. 
2. Ozdogan M, Devay AO, Gurer A et al. Plasma total anti-oxidant capacity correlates inversely with the extent of acute appendicitis: a case control study. World J. Emerg. Surg. (2006)., 24: 1-6.

3. Serefhanoglu K, Taskin A, Turan $\mathrm{H}$ et al. Evaluation of oxidative status in patients with brucellosis. Braz. J. Infect. Dis. (2009). 13(4): 249-251.

4. McCord JM·Human disease, free radicals, and the oxidant/antioxidant balance. Clin Biochem. 1993 Oct;26(5):351-7.

5. Kundi H, Ates I, Kiziltunc $\mathrm{E}$ et al. A novel oxidative stress marker in acute myocardial infarction; thiol/disulphide homeostasis.Am J Emerg Med. 2015 Nov;33(11):1567-71. doi: 10.1016/j.ajem.2015.06.016. Epub 2015 Jun 14.

6. Circu ML, AwTY. Reactive oxygen species, cellular redox systems, and apoptosis. Free Radic Biol Med 2010;48(6):749-62. http://dx.doi.org/10.1016/j.freeradbiomed. 2009.12.022

7. Ozcan Erel, Salim Neselioglu. A novel and automated assay for thiol/disulphide homeostasis. Clinical Biochemistry 47 (2014) 326-332. DOI: 10.1016/j.clinbiochem.2014.09.026

8. Wudkowska A, Goch J, Goch A. Ischemia-modified albumin in differential diagnosis of acute coronary syndrome without ST elevation and unstable anginapectoris. Kardiologia Polska 2010; 68(4): 431-7.

9. Aran T, Unsal MA, Güven S et al. Carbon dioxide Pneumoperitoneum Induces Systemic Oxidative Stres: a clinical study. Eur J Obstet Gynecol Reprod Biol 2012; 161(1): 80-3.

10. Ma SG, Wei CL, Hong B et al. Ischemia-modified albumin in type 2 diabetic patients with and without peripheral arterial disease. Clinics 2011; 66(10):1677-80.

11. Mastella AK, Moresco RN, da Silva DB et al. Evaluation of ischemiamodified albumin in myocardial infarction and prostatic diseases. Biomed Pharmacother 2009; 63.762-6.

12. Lippi G, Montagnana M; Ischemia Modified Albumin in Ischemic Disorders. Ann Thorac Cardiovasc Surg 2009; 15(2): 137

13. Krauss BS, Harakal T, Fleisher GR. General trauma in a pediatric emergency department: spectrum and consultation patterns. Pediatr Emerg Care. 1993;9(3):134

14. Kazez A. Trauma in children. Turk Arch Ped 2010; 45 Suppl: 12-6

15. Mikrogianakis A. Penetrating Abdominal Trauma in Children. Clinical Pediatric Emergency Medicine. 2010;11(3): 217-224. DOI: http://dx.doi.org/10.1016/j.cpem.2010.06.006

16. Bambini DA, Almond PS. Abdominal trauma. In: Arensman RM, Bambini DA, Almond PS, Adolph V, Radhakrishnan J. Pediatric Surgery (second edition). Austin, Texas, USA: Landes Bioscience, 2009: 128-31.

17. Alam S, Abrahamsson K, Lewis FC, Radhakrishnan J. Genitourinary trauma. In: Arensman RM, Bambini DA, Almond PS, Adolph V, Radhakrishnan J. Pediatric Surgery (second edition). Austin, Texas, USA: Landes Bioscience, 2009: 132-5.

18. Turan C, Okur H, Bekerecioğlu A et al. Çocuklarda toraks travmaları. T Klin Pediatr 1994; 3: 48-51

19. Nayak CD, Nayak DM, Raja A et al. Erythrocyte indicators of oxidative changes in patients with graded traumatic head injury. Neurol India. 2008; 56:31-35

20. Aslan A, Gurelik M, Cemek M et al. Nimodipine can diminish oxidative stress in patients with severe head trauma. J Neurosurg Sci. 2012 Sep;56(3):247-53.

21. Tyurin VA1, Tyurina YY, Borisenko GG et al. Oxidative stress following traumatic brain injury in rats: quantitation of biomarkers and detection of free radical intermediates. J Neurochem. 2000 Nov;75(5):2178-89.

22. Ana Rodriguez-Rodriguez, Juan Jose Egea-Guerrero, Francisco Murillo-Cabezas et al. Oxidative Stress in Traumatic Brain Injury. Curr Med Chem. 2014 Apr;21(10):1201-11.

23. Parihar A, Parihar MS, Milner S et al. Oxidative stress and anti-oxidative mobilization in burn injury. Burns, 2008; 34: 6-27.

24. Sheweita SA, Khoshhal KI. Calcium metabolism and oxidative stress in bone fractures: role of antioxidants. Curr Drug Metab. 2007 Jun;8(5):519-25.

25. Arauz J, Ramos-Tovar E, Muriel P. Redox state and methods to evaluate oxidative stress in liver damage: From bench to bedside. Ann Hepatol. 2016 Mar-Apr;15(2):160-73. doi: $10.5604 / 16652681.1193701$. 
26. Malek M, Nematbakhsh M. Renal ischemia/reperfusion injury; from pathophysiology to treatment. Journal of Renal Injury Prevention. 2015;4(2):20-27. doi:10.12861/jrip.2015.06.

27. Bedreag HO, Rogobete AF, Sarandan M et al. Oxidative stress in severe pulmonary trauma in critical ill patients. Antioxidant therapy in patients with multiple trauma-a review. Anaesthesiol Intensive Ther 2015; 47(4): 351-359.

28. Li R, Chen B, Wang G et al. Effects of mechanical strain on oxygen free radical system in bone marrow mesenchymal stem cells from children. Injury, Int. J. Care Injured 42 (2011) 753-757

29. Bar-Or D, Lau E, Winkler JV. A novel assay for cobalt-albumin binding and its potential as a marker for myocardial ischemia a preliminary report. J Emerg Med 2000; 19:311-5.

30. Yıldırım A, Aslan Ş, Ocak T et al. Serum paraoxonase/arylesterase activities and malondialdehyde levels in trauma patients. Eurasian J Med. 2007;39(2):85-8.

31. Rana SV, Kashinath D, Singh G et al. Study on oxidative stress in patients with abdominal trauma. Mol Cell Biochem. 2006; 291(1-2):161-6.

32. Velioglu S. Effects of natural antioxidants on human health. Gida. 2000; 25 (3):167-76.

33. Rael LT, Bar-Or R, Aumann RM et al. Oxidation reduction potential and paraoxonase-arylesterase activity in trauma patients. Biochem Biophys Res Commun. 2007; 361(2):561-5.

34. Buyukaslan H, Gulacti U, Gökdemir MT et al. Serum thiol levels and thiol/disulphide homeostasis in gunshot injuries. Euro J of Trauma and Emerg Surgery https://doi.org/10.1007/s00068-017-0900-9

35. Giden R, Gokdemir MT, Erel O et al. The Relationship Between Serum Thiol Levels and Thiol/Disulfide Homeostasis with Head Trauma in Children. Clinical Laboratory 2018; 64(1):163-168.

Table 1. The frequency of the systems or organs that has been affected by trauma

\begin{tabular}{ll}
\hline Systems and organs affected & $\mathrm{n}$ \\
\hline Spleen & 37 \\
Liver & 34 \\
Lungs & 33 \\
Extremities & 17 \\
Kidney & 12 \\
Central nervous system & 10 \\
Intestinal system & 8 \\
Pancreas & 1 \\
Bladder & 1 \\
\hline
\end{tabular}

$\mathrm{n}$ : frequency

Table 2. The comparison of laboratory findings of the variables in the groups

\begin{tabular}{lllllllll}
\hline & Groups & $\mathbf{n}$ & Mean & Median & SD & Min & Max & $\mathbf{p}^{*}$ \\
\hline Variables & & & & & & & & \\
Native thiol (SH) & Trauma & 101 & 323.89 & 324.2 & 78.09 & 125.6 & 504.7 & 0.000 \\
& Control & 101 & 404.93 & 399.1 & 66.28 & 259.3 & 673.2 & \\
Total thiol (SH+SS) & Trauma & 101 & 368.45 & 369.1 & 81.84 & 150.5 & 565.2 & 0.000 \\
& Control & 101 & 444.18 & 369.68 & 72.81 & 279.6 & 742.5 & \\
Dynamic disulphide (SS) & Trauma & 101 & 22.22 & 21 & 7.97 & 4 & 52 & 0.001 \\
& Control & 101 & 19.64 & 19 & 4.8 & 10 & 35 & \\
Dynamic disulphide / Total thiol, \% & Trauma & 101 & 0.06 & 0.05 & 0.024 & 0.01 & 0.18 & 0.000 \\
& Control & 101 & 0.044 & 0.043 & 0.008 & 0.03 & 0.08 & \\
Ischemia-modified Albumin (IMA) & Trauma & 101 & 0.49 & 0.47 & 0.22 & 0.14 & 1.74 & 0.000 \\
& Control & 101 & 0.63 & 0.65 & 0.17 & 0.27 & 1.32 & \\
Albumin & Trauma & 101 & 4.31 & 4.4 & 0.38 & 2.5 & 5 & 0.000
\end{tabular}




\begin{tabular}{lllllllll}
\hline Groups & $\mathbf{n}$ & Mean & Median & SD & Min & Max & $\mathbf{p}^{*}$ \\
\hline Control & 101 & 4.5 & 4.5 & 0.19 & 3.7 & 6 & \\
\hline
\end{tabular}

n: frequency, SD: standard deviation, Min: Minimum, Max: Maximum, *: Mann Whitney U test

Table 3. The comparison of the trauma sub-groups' laboratory findings

\begin{tabular}{llllll}
\hline & Groups & $\mathbf{n}$ & Mean & SD & p* \\
\hline Variables & & & & & \\
Native thiol (SH) & Surgery & 17 & 317.1235 & 76.61708 & 0.61 \\
\multirow{2}{*}{ Total thiol (SH+SS) } & Follow-up & 84 & 312.3574 & 64.22339 & \\
\multirow{2}{*}{ Dynamic disulphide (SS) } & Surgery & 17 & 4.4235 & .23326 & 0.57 \\
& Follow-up & 84 & 4.2964 & .38976 & \\
Dynamic disulphide / Total thiol, \% & Surgery & 17 & 23.8824 & 10.01176 & 0.51 \\
& Follow-up & 84 & 21.6429 & 7.49882 & \\
Ischemia-modified Albumin (IMA) & 17 & .0631 & .02397 & 0.73 \\
& Follow-up & 84 & .0622 & .02415 & \\
Albumin & Surgery & 17 & 0.4622 & 0.1477 & 0.67 \\
& Follow-up & 84 & 0.5102 & 0.26865 & \\
& Surgery & 17 & 364.8588 & 77.71375 & 0.62 \\
& Follow-up & 84 & 355.7327 & 68.63171 & \\
\hline
\end{tabular}

n: frequency, SD: standard deviation, *: Mann Whitney U test

Table 4. The comparisons of the laboratory findings in terms of trauma type

\begin{tabular}{llllll}
\hline & Groups & $\mathbf{n}$ & Mean & SD & $\mathbf{p}^{*}$ \\
\hline Variables & & & & & \\
Native thiol (SH) & Penetrating & 15 & 322.79 & 55.16 & 0.65 \\
& Blunt & 86 & 311.48 & 67.94 & \\
Total thiol (SH+SS) & Penetrating & 15 & 364.78 & 61.33 & 0.78 \\
& Blunt & 86 & 355.96 & 71.55 & \\
Dynamic disulphide (SS) & Penetrating & 15 & 21.13 & 8.75 & 0.41 \\
& Blunt & 86 & 22.17 & 7.86 & \\
Dynamic disulphide / Total thiol, \% & Penetrating & 15 & 0.06 & 0.01 & 0.70 \\
& Blunt & 86 & 0.06 & 0.03 & \\
Ischemia-modified Albumin (IMA) & Penetrating & 15 & 0.47 & 0.12 & 0.98 \\
& Blunt & 86 & 0.51 & 0.27 & \\
Albumin & Penetrating & 15 & 4.39 & 0.22 & 0.14 \\
& Blunt & 86 & 4.31 & 0.39 & \\
\hline
\end{tabular}

$\mathrm{n}$ : frequency, SD: standard deviation, *: Mann Whitney U test

\section{Hosted file}

Tables trauma.pdf available at https://authorea.com/users/393813/articles/507362-evaluationof-dynamic-thiol-disulfide-balance-and- $i \% C C \% 87$ schemia-modified-albumin-in-children-withtrauma 